\title{
Laboratory testing under managed care dominance in the USA
}

Y Takemura, J R Beck

\begin{abstract}
The uncontrolled escalation of total health care expenditure despite the government's endeavours during the past decades in the USA had led to the rapid infiltration of managed care organisations (MCOs). Traditional hospital based laboratories have been placed in a crucial situation with the advent of the managed care era. A massive reduction of in house testing urged them to develop strategies against financial difficulty. Consolidation and networking, participation in the outreach testing market, and emphasis on point of care/satellite laboratory testing in nontraditional, ambulatory settings are major strategies for the survival of hospital laboratories. Several physicians' office laboratories (POLS) have closed their doors in response both to regulatory restrictions imposed by the Clinical Laboratory Improvement Amendments of 1988 and to managed care infiltration. It seems likely that POLs and hospital laboratories will continue to reduce test volumes, whereas commercial reference laboratories will thrive through contracting with MCOs. In the current climate of managed care dominance in the USA, clinical laboratories are changing their basic operation focus and mission in response to the aggressively changing landscape. (f Clin Pathol 2001;54:89-95)
\end{abstract}

Keywords: laboratory testing; managed care organisations; survival strategies

Health care has been the world's most successful industry, nowhere more so than in the USA. The prosperity of the health care industry has been accompanied with tremendous expenses in this country. The government's efforts to slow the rapid growth of USA health care expenditure were not effective, and this led to a rapid and progressive penetration of managed care plans in the early 1990s. The medical "cottage industry" in which the patient was the focus of the medical professionals' attention and endeavours is being replaced by the corporate management of many health care activities in which financial profits are being given first priority. ${ }^{1}$ Competitive market principles introduced by managed care business have changed the mission of many medical services, including laboratory testing. Clinical laboratories, especially the large commercial variety, act more and more like a utility company and the laboratory test is viewed increasingly as a commodity. ${ }^{2}$ Traditional hospital based laboratories, which were placed in an extremely critical situation, owing to a massive decrease of in house tests, had to respond to financial difficulty by developing strategies for survival. Consolidation and networking of intrahospital or interhospital laboratories, successful outreach programmes, and "point of care" testing in outpatient based, non-traditional settings are keys for the survival of hospital laboratories in the managed care environment.

This article is a documentation of recent changes mainly occurring in clinical laboratories belonging to clinical pathology or laboratory medicine disciplines in the USA.

\section{Rapid escalation of USA health care} expenditure under the "fee for service" reimbursement practice

Total health care expenditure in the USA has rapidly grown under the fee for service reimbursement practice, which dates from the establishment of Medicare/Medicaid programmes in 1965. Between 1960 and 1980, the total annual expenses for health care in this country increased from US $\$ 26.9$ billion to $\$ 247.2$ billion/year, and this represented a change from $5.1 \%$ to $8.9 \%$ of the gross domestic product (GDP). ${ }^{3}$ Factors such as the development of new technology, increasing physician supply, and a growing elderly population, as well as financial incentives for hospitals and physicians under the open ended reimbursement system, generated a rapid expansion of personal health care costs during this period. ${ }^{4}$ The costs of clinical laboratory services contribute to the general inflation in medical and health care costs so that there is a heightened concern about regulating clinical laboratories and controlling unnecessary costs associated with laboratory testing. ${ }^{5}$ Increases in laboratory expenditure were facilitated by the disparity between laboratory charges and costs because laboratory pricing policies were not related solely to real costs or services offered. ${ }^{6-8}$ The price/cost disparity has promoted test utilisation, and resulted in the clinical laboratory becoming a "profit centre" for hospital budgeting.

The provision and costs of health care have become national issues in the USA since the 1970s. Medicare (federal funding)/Medicaid programmes (state and federal funding), which provide a health insurance for aged $(\geqslant 65$ years old) and low income individuals, respectively, have been accompanied by a tremendous increase in expenses: Medicare costs exceeded US $\$ 70$ billion in 1985 , a more than 10 -fold increase since its enactment, and Medicaid expenditure had also increased, reaching approximately $\$ 40$ billion in 1985 (the Health 
Care Financing Administration (HCFA), national health care expenditure database). Rapid escalation of federal health care expenditure led to a consensus that the post-Medicare era resulted in greater spending, with minimal incentives to improve hospital efficacy, and that increased productivity served only to diminish cost based, third party payer reimbursement unless utilisation controls were achieved. ${ }^{5}$ Congress focused on the retrospective, fee for service reimbursement system, which had been adopted since the establishment of Medicare for the growth containment of its expenditures in the payment reform manoeuvres of the early 1980s. ${ }^{9}$

\section{The government's attempt to reform the Medicare reimbursement system in the early 1980s}

The government's efforts at Medicare reform had led to legislation of the prospective payment system (PPS) based on the diagnosis related groups (DRG) for Medicare inpatient reimbursement in 1983. The DRG/PPS revolutionised the delivery of health care in the USA. Instead of considering the retrospective cost, Medicare expenses under this new system are reimbursed on a predetermined, cost/case basis, or a PPS, for the diagnosis that has been largely established at the time of a patient's admission. The PPS allows hospitals a simple, predetermined sum to cover all expenses of a given hospitalisation. The single DRG fee creates powerful incentives to reduce unnecessary services, shorten lengths of hospital stay, and decrease admissions. Whereas a cost based, retrospective reimbursement system provides little incentive to contain costs, and physicians generate additional revenues by providing more complex services, a prospective reimbursement based on the DRG contains an incentive for cost containment. ${ }^{5}$ The introduction of the DRG/PPS system to Medicare inpatient reimbursement has converted hospital laboratories from the position of "profit centre" under the fee for service reimbursement system to the "cost centre" funded by hospital income, at least for inpatient laboratory testing. Hospital income from Medicare inpatients is independent of laboratory test volume and complexity in the new system. Hospitals have reduced laboratory operating expenses by constraining laboratory growth and development. More than $70 \%$ of laboratories have undergone budget reductions. ${ }^{10}$

However, the economic pressure on hospitals and physicians has not achieved much success because services have simply been shifted to outpatient facilities and non-hospital settings, such as private physicians' offices, which were less regulated and paid on a cost reimbursed basis. In fact, outpatient services have grown throughout the 1980 s because hospitals were forced to shorten the length of hospital stays owing to financial incentives under the predetermined, fixed fee reimbursement system. In addition, technological advances allowed more care to be provided outside the hospitals. For example, over $40 \%$ of hospital surgical procedures were being per- formed on an outpatient basis. Declining hospital use under the PPS was also related to increases in the use of skilled nursing facilities, intermediate care nursing homes, and home health care agency visits, as well as a very rapid growth in cost reimbursed psychiatric and rehabilitation facilities. ${ }^{11}$ Similarly, hospitals have shifted diagnostic testing from inpatient to outpatient setting, where a separate reimbursement paid by a reasonable cost basis is allowable, including preadmission testing. Compensating for inpatient losses, the hospital laboratories profited from the testing that was associated with the care in outpatient settings. There was little competition for this newly captured business, because commercial laboratories were effectively barred from this outpatient laboratory test market by the integration of testing with the site of service. ${ }^{12}$

The PPS in which the DRG is the basic unit of payment could not stop ongoing trends in clinical laboratory testing dating from the 1960s, particularly in large hospitals such as tertiary care, academic medical centres. The expanded growth in laboratory test volume between 1960 and 1990 was supported by automation, computerisation, and technological advances, with an estimated growth rate of approximately $15 \%$ /year in the 1970 s and $12 \%$ in the $1980 \mathrm{~s}$ in an academic medical centre. ${ }^{13} 14$ This annual growth resulted from the availability of new tests, increased use of existing tests, increased test volume as a result of new treatment programmes such as organ transplantation, additional hospital beds in special units such as intensive care, and expanded outpatient services. There was no convincing evidence that the introduction of the DRG/ PPS appreciably changed this trend in laboratory testing. ${ }^{14}$

Hospitals benefited from the use of increasingly sophisticated patient care in the late 1980s. The government's attempt to curb medical costs through the fixed fee reimbursement system for Medicare inpatients failed because of the growth of outpatient services and the inability to control patient admissions. Again, there were no real incentives to influence physician and hospital behaviour (the prospective payment assessment commission report, 1994), and thus, national health care expenses grew rapidly even after the tremendous achievement for the Medicare/Medicaid payment system in the early 1980 s.

The implementation of the DRG/PPS for Medicare reimbursement to hospitals system was a revolution in the delivery of health care in the USA. However, DRG induced changes had a limited impact on slowing the growth of total national health care expenditure or laboratory related expenditure, although this tremendous achievement had important effects, such as shorter lengths of hospital stay and a decrease in admission. This may be attributable to the narrowly focused government policy on constraining the Medicare/Medicaid budget growth alone rather than a comprehensive, systematic health care policy. ${ }^{11}$ Nevertheless, it should be emphasised that the prospective, fixed fee reimbursement system brought a 
heightened concern regarding the control of unnecessary costs, or cost effective resource utilisation. The revolution in the public sector of health insurance has been followed by the competitive, private insurance industry, which has begun to formulate its own cost containment programmes. Cost saving consciousness arising in the third party payers of private health insurance and in employers also allowed the gradual increase of managed care organisations (MCOs) during the 1980s. ${ }^{91516}$

\section{Rapid and aggressive spread of MCOs in the 1990s}

Total health care expenditure in the USA grew exponentially during the late 1980s and early $1990 \mathrm{~s}$, and was anticipated to reach almost a trillion dollars in 1995 (13.6\% of the GDP). ${ }^{3}$ The government had attempted to enact new legislation to constrain the expansion of health care costs in 1993, proposing an employer based, federally subsidised programme. ${ }^{17} 18$ However, this proposal was widely opposed, particularly by the insurance industry and employers of small business firms, and was turned down by Congress. Subsequently, federal initiatives in health care reform have fallen from prominence and, instead, MCOs have taken over. Employers are switching health insurance benefits for their employees from conventional, indemnity plans to managed care plans, and consequently, managed care covered nearly $75 \%$ of insured working Americans by $1995 .{ }^{19} 20$

Managed care is a coordinated plan to provide health care of high quality at a lower cost. In theory, costs are lowered by cost sharing with beneficiaries; establishing incentives for providing patient care in outpatient settings, reducing hospital admissions, and shortening lengths of hospital stay; selectively contracting with health care providers; and directly managing high cost medical care cases. ${ }^{1}$ In other words, spending has been slowed in three ways under managed care: reducing services to patients, providing services more efficiently, and squeezing the incomes of physicians and other health care professionals. ${ }^{21}$ Providers are often reimbursed on a prepaid monthly basis (capitation) for all medical services as a means of controlling costs and fostering incentives that can change physician's behaviour. One of the basic management tenets for managed care is to place all financial risk, or as much as possible, on the providers (physicians and hospitals) most directly by capitation. ${ }^{22}$ In recompense for lowered insurance fees, MCO members (patients) receive fewer medical services and have restrictions imposed by MCOs: for example, the Health Maintenance Organisation (HMO), a classic MCO, does not usually allow its enrollees to be cared for by out of network physicians or by specialists without a referral from his/her primary care physician (so called, "gatekeeper"). Voluntary access to those other than a primary care physician whom an enrollee chooses is not allowed at the initial practice. Financial incentives encourage the gatekeeper to limit medical services to those considered essential. Competitive market principles under a managed care dominance, however, led to appreciable success in restraining USA health care costs. ${ }^{23}$

\section{The effects of managed care on hospital} based laboratories

Managed care has had profound effects on clinical laboratories in the USA. The most important tenet of MCOs is the elimination of unnecessary medical care/resource use and, thus, the overuse of diagnostic tests is strictly discouraged. In addition, new businesses in a managed care environment such as surgicentres, urgent care centres, sophisticated $x$ ray centres, and diagnostic clinics, which are independent of hospitals and frequently run by MCOs, affected traditional hospital laboratories adversely. Comparing the market share of laboratory testing in the USA in 1986 with that in 1996, tests performed in hospital laboratories declined from $52 \%$ to $46 \%$, whereas the share of commercial reference laboratories (CRLs) expanded to almost double, reaching $39 \%$ of all laboratories by $1996 .{ }^{24}{ }^{25}$

Once managed care reaches $30-40 \%$ in a community, it begins to exert a considerable force against the health care delivery system. ${ }^{26}$ At this level, MCOs can direct their members (patients) to specific hospitals and physicians who contract with MCOs, and demand substantial discounts in most medical care processes. Shorter inpatient stay is strongly encouraged, resulting in "quicker and sicker" discharges of patients. They discourage hospital admission, and shift the centre of patient care away from the hospital and into outpatient environments. Traditionally, hospital laboratories have focused on inpatient testing, emphasising high technical quality, rapid turnaround time for acutely ill patients, collaboration with clinicians in providing patient care, and quality improvement and accreditation. This basic mission of hospital laboratories has led to a high unit cost for each test when compared with testing in commercial laboratories. ${ }^{27}$ Shorter hospital stays do not necessarily generate less testing, but fewer admissions most certainly do. In addition, preadmission testing is often performed in non-hospital laboratories. ${ }^{12}$ Managed care has also discouraged consultations with specialists. MCOs carefully direct the use of services when allowing a patient admission, and the use of high cost services is also controlled. Therefore, the net result of the influence of managed care has been a massive reduction of in house tests, particularly on inpatients. In response to the progressive infiltration of MCOs, most hospital laboratories have aggressively cut costs by reducing staff numbers and consolidating or sharing services with other laboratories. ${ }^{27}$

With the shift of patients to outpatient facilities, hospitals gained more revenue from this setting, which compensated in part for hospital losses in inpatient care before the advent of the managed care era. However, competition for patients is becoming more intense as numerous "for profit" providers have sprung up to care for patients on an outpatient basis. The new 
providers are a product of the managed care business; examples include: surgicentres, free standing magnetic resonance imaging (MRI) and computed tomography (CT) centres, home care agencies, home infusion enterprises, and rehabilitation services, all of which take business from the hospitals that traditionally provided these services. Compared with these new providers, hospitals have traditionally been high cost providers of services, particularly ancillary services such as laboratory and $x$ ray tests, so they have great difficulty competing in the new environment. ${ }^{12}$ These new businesses have deprived hospital laboratories of outpatient testing. For example, emergency room testing is currently a profitable business for many hospitals; however, the growth of urgent care centres run by MCOs threatens the long term prospects of this revenue source. In addition, restrictions on payments by MCOs limit the ability of physicians to refer patients to the high cost hospital laboratory. Both cost and payment considerations move more outpatient testing from hospital laboratories into CRLs.

\section{Strategies for survival of hospital based laboratories in a managed care environment}

In the extremely crucial situation in which hospital based laboratories are currently placed under the managed care environment, clinical laboratories are strongly urged to change their operation focus and to develop strategies for survival. These include the consolidation and networking of laboratories, participation in the outreach testing market, the development of stronger outpatient departments, the reacquisition of outpatient testing, and an emphasis on point of care/satellite laboratory testing.

CONSOLIDATION AND NETWORKING OF HOSPITAL BASED LABORATORIES

During the past decade, laboratory testing performed in testing sites outside the main centralised clinical laboratory in a hospital has been growing because of the importance of timely diagnostic results obtained within the patient care sites. ${ }^{28-30}$ Because decentralisation of laboratory testing must result in lower cost efficiency, hospitals are attempting the recentralisation of in house testing sites and the consolidation of operating units within a laboratory to reduce laboratory operating costs. ${ }^{31-33}$ In addition, the costs of performing laboratory tests in one core facility are often lower than the alternative of performing the same tests in individual hospital laboratories, mainly because of the increased test volume. ${ }^{34}$ Furthermore, the duplication of laboratory services can be reduced, by forming a strategic alliance of hospital based laboratories in the same geographical region. Moreover, external laboratory consolidation, in which allied hospital laboratories share services or cooperate with each other at least at some level is beneficial not only for lowering costs/test but also for entering the outreach testing market. In this context, hospital laboratory administrators have come to consider the consolidated laboratory network (CLN) as an effective strategy for the survival of hospital based laboratories under the steep cost pressures imposed by MCOs. In fact, advances in automation, novel robot based technology, and the network architecture of laboratory information systems (LIS) have allowed the vision of a CLN to become real. ${ }^{34}$ CLN is a system in which many of the non-urgent laboratory tests that in the past were performed in separate hospital laboratories are now being sent to a core laboratory. Regional laboratories in a network are functioning as "urgent test" laboratories only for time dependent tests. By integrating and restructuring hospital laboratories with this approach, some have developed more aggressive outreach programmes, successfully participating in the reference laboratory market. ${ }^{27}{ }^{34-37}$

\section{PARTICIPATION IN THE OUTREACH TESTING} MARKET

Clinical laboratory service/diagnostic testing has come to be viewed primarily as a business and a commodity rather than a profession and a medical service in the current climate of managed care dominance. ${ }^{12}$ Laboratories that produce reliable results at a lower cost are more attractive to managers not trained in science or medicine. The rapid growth of CRLs under managed care, which considers that price rather than quality is the key to success, facilitates the commercialisation of laboratory services. It has become clear that to improve financial performance and to survive, hospital based laboratories have to widen their patient base by entering the reference laboratory market with the development of successful outreach programmes. They have done this in three ways. ${ }^{24}$ (1) By forming networks and developing outreach programmes, thereby competing with CRLs for outreach testing referred from independent facilities and office physicians. (2) By forming coalitions with reference laboratories. This partnership structure leads to increased productivity in hospital laboratories, merging hospital inpatient work with outreach work collected by reference laboratories. ${ }^{38} 39$ The reference laboratories gain from this partnership by sharing pathologists and specialists in the allied hospitals as advisors. (3) By becoming full service reference laboratories themselves with independent associations with pre-existing CRLs. By analysing the arrival times of specimens and reorganising laboratory instruments to accommodate a higher volume of tests, the transformation from a general hospital laboratory into a hybrid combination of inpatient and outreach work can be achieved successfully, with profit from the additional reference testing. ${ }^{40}$

REACQUiSITION OF OUTPATIENT TESTING, DEVELOPING STRONGER OUTPATIENT DEPARTMENTS, AND POINT OF CARE/SATELLITE LABORATORY TESTING IN NON-TRADITIONAL SETTINGS

Hospital based laboratories had been sheltered to a large extent from the competitive pressures of the clinical laboratory industry. Accordingly, with the advent of the highly competitive, managed care era, hospitals have lost not only inpa- 
tient testing but also the testing and profit associated with outpatient care. Newly developed, independent ambulatory centres such as surgicentres, urgent care centres, sophisticated $x$ ray centres, diagnostic clinics, and rehabilitation centres prefer to contract with low cost commercial providers for routine, non-urgent tests. Thus, hospital laboratories are unable to profit from ambulatory care business, despite the shift of patients into outpatient facilities. These centres may be inpatient facilities. Small hospitals in suburban or urban areas probably will cease to exist or will be merged into larger institutions. Those that cease to exist may either be replaced by outpatient facilities or, in many cases, might become providers of outpatient care themselves. ${ }^{12}$ These outpatient facilities will not require full service laboratories: routine, non-urgent tests will be sent out to CRLs; they will provide only the results of time dependent tests through small on site laboratories in the manner of point of care testing (POCT). Successful hospitals have responded to the influences of managed care by developing stronger outpatient departments in their hospitals. Even in community hospitals, strengthened outpatient departments such as cancer centres and sophisticated $x$ ray centres can now be found, and as outpatient visits to these hospital ambulatory centres have increased, outpatient laboratory testing has increased proportionally. ${ }^{24}$ Testing associated with these centres might ultimately be performed as POCT: in this context, many hospital laboratories have become urgent test laboratories, a very different model from the full service hospital laboratories of the past. Because immediate availability of test results in this setting must contribute to patient satisfaction and will result in improved patient outcomes and subsequently in cost effective patient care $^{41}$ others also emphasise the importance of POCT as a survival strategy for hospital based laboratories. ${ }^{42}{ }^{43}$ Certainly, a large market will exist for immediate testing services (which reference laboratories will be unable to provide) in the outpatient environment as more and more complex care is provided in this setting.

\section{Physicians' office laboratories (POLs) under the Clinical Laboratory Improvement Amendments of $\mathbf{1 9 8 8}$ (CLIA 88) and in the managed care environment}

As a result of the PPS, laboratory testing has moved into more outpatient and non-hospital settings. The volume and breadth of tests in POLs and their expenditure have increased since the passage of the DRG/PPS legislation by Congress in 1983. This growth is also supported by technological advances that either reduce the size and cost of equipment necessary to perform tests on an automated basis, or replace complex testing methods requiring highly trained personnel with simple to use, inexpensive test kits. ${ }^{44}$ Although large laboratories in hospitals and CRLs were federally regulated, and operated under guidelines promulgated by the Clinical Laboratory Improvement
Amendments of 1967 (CLIA 67), POLs were exempt from these regulations, causing problems of lower accuracy of laboratory work, more errant results, and less well educated personnel compared with licensed, large hospital and commercial laboratories. ${ }^{45-49}$ The lower quality of laboratory testing in unlicensed POLs led to the CLIA 88 legislation, which extended laboratory regulations to all laboratories regardless of size, complexity, or location. According to the implementation of the CLIA 88 in 1992, all testing sites including POLs must have inspections by a state agency acting on behalf of the HCFA and a certificate issued by the Department of Health and Human Service (HHS) for each category of tests that are to be performed. The basic requirements of CLIA 88 for clinical laboratories in each testing site cover the full spectrum of clinical laboratory functions, such as: (1) personnel qualification; (2) patient test management; (3) facilities, equipment, and supplies; (4) quality control; (5) quality assurance; (6) record keeping; and (7) proficiency testing. The CLIA 88 legislation has improved the quality of testing in POLs, forcing office physicians to deal with the problem of laboratory quality management, thereby increasing laboratory costs. Failure at inspection is followed by large sanctions that threaten the finances of the POLs and may lead to laboratory closure. ${ }^{3}$ Thus, compliance with the CLIA 88 standards is expensive.

Recent studies have demonstrated that on site testing in POLs has been reduced, discontinued, or changed as a result of the CLIA 88 legislation..$^{51}$ Nationally, at a rough estimate, 12500 POLs have closed since 1988 (the total number of POLs functioning is nearly 87000 , according to the HCFA). ${ }^{24}$ In addition, physicians have restricted test menus because regulations require inspections for tests with higher complexity methodology, but not for waived tests alone or for physician performed microscopy (PPM) status. ${ }^{52}$ Now, only $40 \%$ of POLs perform moderate or high complexity tests, which are considered sophisticated enough to require laboratory inspection under CLIA $88 .^{24}$

The market share of POLs has dropped from $28 \%$ in 1986 to $15 \%$ of all laboratories in $1996^{25}$; however, this fall has been largely caused by the progressive dominance of managed care rather than by the legislation of CLIA 88. MCOs understand that the growth of POLs was based on the financial incentives for office physicians under the fee for service reimbursement practice, and fear high test frequency (overuse of laboratory tests) in their own offices. Therefore, MCOs often refuse to negotiate with physicians about laboratory tests performed in their offices and limit reimbursement to a very few in house procedures, preferring all tests to be sent out to CRLs that offer tests at a lower price on the basis of a contract with an $\mathrm{MCO}^{24}$ Again, POLs are performing more waived and PPM tests, which are simple and have low costs, and fewer moderate and high complexity tests. MCOs did not consider the policy of "diagnostic therapeutic process optimisation", which would result in improved 
patient outcome and ultimately lead to cost effective patient care, with on site testing in POLs.

Managed care is a major threat for all physicians' laboratories irrespective of whether they are large or small. Because of the basic tenet of the MCOs that tests are costly, they encourage and often request many tests formerly done in the POLs to be done in CRLs. ${ }^{24}$ To reverse this trend, office physicians must make their laboratories attractive to MCOs by emphasising quality, promoting customer satisfaction, discussing cost effectiveness, and discounting testing fees. ${ }^{53}$

\section{Growing market share of CRLs}

Before implementation of the DRG/PPS, approximately half of laboratory expenditures were generated by hospital based laboratories, with the remainder generated in approximately equal proportions by CRLs and POLs. ${ }^{54}$ During recent years (1986 to 1996), hospital laboratories and POLs have lost part of their share in the laboratory testing market, whereas CRLs have almost doubled theirs. ${ }^{25}$ Because several POLs closed in response to the CLIA 88 restrictions, more tests were sent to reference laboratories as outreach tests. In addition, many of the moderately and highly complex tests formerly done by POLs are now performed by reference laboratories. Furthermore, MCOs limit reimbursement to a few in house procedures in physician's offices. Rapidly growing independent ambulatory centres often run by MCOs deprive hospital outpatient departments of profitable outpatients and testing, and laboratory tests in this setting flow into the reference laboratory market. In an extreme example, some HMOs that have enrolled employees of academic medical centres require all laboratory testing to be sent to large for profit testing facilities. ${ }^{1}$ Indeed, MCOs view CRLs, which produce reliable results at the least expensive prices, as their attractive partner. Thus, managed care has definitely induced the massive growth of independent, for profit reference laboratories.

CRLs provide comprehensive services to hospitals and office physicians, even offering phlebotomy services. Specimens are collected during the day and transported to the laboratory in the evening for analysis at night. Results are returned via computer or fax by the next morning. Reducing costs for, and prices of, tests by capitalising on bulk purchasing and large test volumes, this commercial sector in the laboratory testing industry has bid successfully to MCOs. ${ }^{1}$ In addition, CRLs can expand their areas of expertise to include informatics because of the high test numbers they are handling: they are able to analyse the numbers of tests ordered by each physician and the proportions of abnormal results for each physician, and share that information with the physicians and an MCO. ${ }^{24}$ This is attractive to MCOs, which attempt cost reduction through managing test usage. ${ }^{55}$

There are two major national, full service reference laboratories at present: Quest Diagnostics (previously Corning Life Sciences and
SmithKline Beecham) and LabCorp (previously Roche and NHL) control approximately $50 \%$ of the commercial laboratory market. Both laboratories have grown in the past few years, mainly by merger and acquisition, rather than by recruiting new customers. ${ }^{2}$ Such industry consolidation can diminish cost pressures, increase purchasing power, and strengthen the remaining players' ability to negotiate capitated contracts with managed care providers. MCOs have been successful in negotiating capitation agreements with reference laboratories similar to the agreements they have with physicians. A "per capita" (per subscriber) contract imposed by MCOs is essentially independent of which test is actually ordered. The shift in financial risk to service providers through a capitated, prospective payment structure, in which providers are prepaid a fixed fee for each plan member covered at a "per member per month rate", regardless of how many services are performed, has increased the financial pressure on providers. In a move to increase profitability, some commercial laboratories are avoiding highly capitated managed care contracts. ${ }^{55}$ Although managed care has helped CRLs to expand their market share, they must contend with discount pricing in the fee for service or financial risk in the capitated structure imposed by MCOs.

\section{Conclusion}

The uncontrolled escalation of total health care expenditure despite the government's endeavours during the past decades in the USA had led to the rapid dominance of MCOs. Diagnostic testing is now viewed as a "commodity" rather than a medical service, and laboratories that produce reliable test results at the least expensive prices are attractive to managed care business. Traditional hospital based laboratories have been placed in a crucial situation with the advent of the managed care era: a massive reduction of in house testing has urged them to develop strategies against financial difficulty. Consolidation, networking, and participation in the outreach testing market are major strategies for the survival of hospital laboratories. They also emphasise point of care/satellite laboratory testing in non-traditional, ambulatory settings to restore outpatient testing deprived by the managed care associated new businesses. Several POLs have closed their doors in response to regulatory restrictions imposed by CLIA 88 and managed care infiltration. It seems likely that POLs and hospital laboratories will continue to reduce test volumes, whereas CRLs will thrive through contracting with MCOs. However, they will have to contend with increasing financial pressures imposed by MCOs because, in the future, medical services undoubtedly will be provided on a fixed, prospective payment, or a capitation basis rather than a traditional fee for service structure.

As more patients are covered by managed care, hospitals, physicians, clinical laboratories, and other providers are increasingly focused on improving quality, efficiency, and care while simultaneously reducing cost. ${ }^{55}$ In the current 
climate of managed care dominance in the USA, clinical laboratories are changing their basic operation focus and mission in response to the aggressively changing landscape: this includes commercial concerns in laboratory services. Traditional laboratories that are unwilling to adapt to the new environment will not survive in this country. This work was supported in part by a grant from the Pfizer
Health Research Foundation. The authors thank D Brath at the Methodist Hospital, Houston, USA for his valuable comments on this study.

1 Conn RB, Snyder JW. Changes in the American health care system: crisis in the clinical laboratory. Clin Chim Acta 1997;267:33-49.

2 Statland BE. The commercialization of lab services: ...or, make no mistake about it, lab testing is big business. Medical Laboratory Observers 1995;27:32-7.

3 Takemura Y, Beck JR. The effects of a fixed-fee reimbursement system introduced by the Federal Government on laboratory testing in the United States. Fapanese fournal of Clinical Pathology 1999;47:1-10.

4 Jencks SF, Schieber GJ. Containing US health care costs: what bullet to bite? Health Care Financing Review. Annual Supplement 1991;1-12.

5 Robinson A. Rationale for cost-effective laboratory medicine Clin Microbiol Rev 1994;7:185-99.

6 Bailey RM, Tierney TM, Jr. Costs, service differences, and prices in private clinical laboratories. Milbank Mem Fund $Q$ Health Soc 1974;52:265-89.

7 Conn RB. Clinical laboratories. Profit center, production industry or patient-care resource? N Engl F Med 1978;298: $422-7$.

8 Lundberg GD. Pathology. $7 A M A$ 1979;241:1382-4.

9 Iezzoni LI. The impact of reimbursement changes on doctors' office testing. Clin Lab Med 1986;6:329-43.

10 Statland BE, Brzys K. Evaluating STAT testing alternatives by calculating annual laboratory costs. Chest 1990;97(suppl 5):198S-203S

11 Feinglass J, Holloway JJ. The initial impact of the Medicare prospective payment system on US health care: a review of prospective payment system on US health care:

12 De Cresce RP, Lifshitz MS, Logue LJ. Managed care and hospital laboratory: survival of the fittest. Clin Lab Manage Rev 1994;8:472-83.

13 Benge H, Bodor GS, Younger WA, et al. Impact of managed care on the economics of laboratory operation in an academic medical center. Arch Pathol Lab Med 1997;121. 689-94

14 Benge H, Csako G, Parl FF. A 10-year analysis of "revenues", costs, staffing, and workload in an academic medical center clinical chemistry laboratory. Clin Chen 1993;39:1780-7.

15 Iglehart JK. The American health care system: managed care. N Engl f Med 1992;327:742-7.

16 Miller RH, Luft HS. Managed care plan performance since 1980. FAMA 1994;271:1512-19.

17 Kricka LJ, Parsons D, Coolen RB. Healthcare in the United States and the practice of laboratory medicine. Clin Chim Acta 1997;267:5-32.

18 McDermott J. Evaluating health system reform: the case for a single-payer approach. $\mathcal{F A M A} 1994 ; 271: 782-4$

19 Drake DF. Managed care: a product of market dynamics. 7AMA 1997;277:560-3.

20 Jensen GA, Morrisey MA, Gaffney S, et al. The new dominance of managed care: insurance trends in the $1990 \mathrm{~s}$. nance of managed care: insurance trend
Health Aff (Millwood) 1997;16:125-36.

21 Fuchs VR. Managed care and merger mania. $f A M A$ 1997;277:920-1.

22 Bodenheimer TS, Grumbach K. Capitation or decapitation: keeping your head in changing times. FAMA 1996;276: 1025-31.

23 Levit KR, Lazenby HC, Braden BR, et al. National health expenditures, 1995. Health Care Financing Review 1996;18: $175-214$

24 Benjamin JT. The effect of CLIA ' 88 and managed care on the medical lab market: its impact on POLs, hospital labs, and reference labs. Medical Laboratory Observers 1996;28: 54-8.
25 Gore MJ. Hospital and commercial labs vie for market conrol. In: Weissman D, ed. Washington G-2 reports. Washington, DC. US Government, 1995:5-8.

26 Kiechle FL. Strategies for survival of a hospital central laboratory in the 21 st century. Am Soc Clin Pathol 1998 Fall National Meeting Booklet. Chicago: American Society of Clinical Pathologists: $1-48$

$27 \mathrm{Lu} \mathrm{S}$, Tam L, Sweeney J, et al. Outreach, consolidation, and networking: Columbia's approach to successful integration of laboratory services in California. Clin Lab Manage Rev 1996;10:507-17.

28 Cousar JB, Peters TH. Laboratories in patient-centered units. Clin Lab Med 1994;14:525-38.

29 Handorf CR, McLendon WW. Opportunities and challenges of laboratory testing at alternative sites. $\mathcal{F A M A}$ 1995;274:1308-9.

30 Kost GJ. Guidelines for point-of-care testing: improving patient outcomes. Am f Clin Pathol 1995;104(suppl 1):S111-27.

31 Farwell DC. Hospital laboratory consolidation. Clin Lab Manage Rev 1995;9:411-20.

32 Fattal GA, Tanasijevic MJ, Winkelman JW. Evolution beyond the shared service model of consolidated hospital clinical laboratories. Arch Pathol Lab Med 1995;119:701-5.

33 Ho DKH. Restructuring the hospital lab: the view from Ontario. Medical Laboratory Observers 1996;28:52-6.

34 Statland BE, Berger D. The consolidated hospital laboratory network: an idea whose time has come. Medical Laboratory Observers 1997;29:27-9.

35 Catarella G, Lien J, Searle K. A successful laboratory outreach program. Clin Lab Manage Rev 1994;8:163-6.

36 Pawlick GF, Davidson PL. Implementing complex network laboratory information systems in a health maintenance organization. Clin Lab Manage Rev 1996;10:555-9.

37 Wright LD, Pillinger CL. Networking pathology services: adjusting to managed care. Clin Lab Med 1996;16:227-41.

38 Engel RH, Herring N, Isola JC. An independent laboratory's experience with a hospital partnership. Clin Lab Manage Rev 1995;9:389-93.

39 Mango PD, Triulzi DJ, Burns SA, et al. The reemergence of the hospital-based laboratory. Clin Lab Manage Rev 1996;10:499-504

40 Tiffany DS. A chemistry department in transition: how we became a reference laboratory. Medical Laboratory Observers $1997 ; 29: 24-30$

41 Takemura Y, Ishida H, Inoue Y, et al. Common diagnostic test panels for clinical evaluation of new primary care outpatients in Japan: a cost-effectiveness evaluation. Chm Chem 1999;45:1752-61.

42 Krienitz D. Point-of-care testing and managed carebeneficial partners. Medical Laboratory Observers 1997(September):30-5.

43 Coffman NB. The competitive marketplace. Medical Laboratory Observers 1998;30:52-4.

44 Ortbals DW. Effect of Medicare/Medicaid reimbursement policies on diagnostic methodology in the physician's office. Diagn Microbiol Infect Dis 1986;4:143S-50S.

45 Grayson RT. Effects of regulatory controls on the accuracy of clinical laboratory tests. Fournal of Medical Technology 1984;1:632-7.

46 Boone DJ. Literature review of research related to the Clinical Laboratory Improvement Amendments of 1988. Arch Pathol Lab Med 1992;116:681-93.

47 Crawley R, Belsey R, Brock D, et al. Regulation of physicians-office laboratories: the Idaho experience. $f A M A$ 1986;255:374-82.

48 Belsey R, Baer DM. Proficiency of office microbiology testing. Clin Lab Med 1986;6:345-54.

49 Bloch MJ, Cembrowski GS, Lembises GJ. Longitudinal study of error prevalence in physicians' office laboratories. $7 A M A$ 1988;260:230-5.

50 Roussel PL. Impact of CLIA on physician office laboratories in rural Washington state. 7 Fam Pract 1996;43:249-54.

51 LaBeau KM, Simon M, Steindel S. Clinical laboratory test menu changes in the Pacific Northwest: 1994-1996. Clin Chem 1998;44:833-8.

52 Benjamin JT. Effects of CLIA ' 88 on the physician office aboratory: a survey of midsized pediatric practices before and after CLIA '88. Clin Pediatr 1996;35:125-8.

53 Bachman MA. The physician office laboratory: profitability under managed care. Med Group Manage f 1997;44:28-31.

54 Shaw ST, Jr, Miller JM. Cost-containment and the use of reference laboratories. Clin Lab Med 1985;5:725-52.

55 Cortese L, Kissel E. Competitive and strategic positioning of national clinical reference laboratories in response to managed care. Clin Lab Manage Rev 1996;10:545-50. 